\title{
屢国pubvet
}

https://doi.org/10.31533/pubvet.v16n01a1019.1-7

\section{Recuperação funcional de cães submetidos à reabilitação pós ressecção de cabeça e colo femoral}

\author{
Luisa Villardi Martins ${ }^{1}$, Renata Tesser $\operatorname{Rocha}^{2} \theta$ \\ ${ }^{1}$ Residente do Hospital Veterinário Anhembi Morumbi, Departamento de Reabilitação Animal, São Paulo - SP Brasil. \\ ${ }^{2}$ Professora da Universidade Anhembi Morumbi, Departamento de Medicina Veterinária, São Paulo -SP Brasil. \\ *Autor para correspondência,E-mail: luisa.m.villardi@gmail.com
}

Resumo. O procedimento cirúrgico de ressecção de cabeça e colo femoral é uma técnica usada com frequência para afecções degenerativas e traumáticas que acometem a articulação coxofemoral. No período de pós-operatório cirúrgico a reabilitação é relevante para que o paciente tenha controle da dor, apoio total do membro, melhora da claudicação e ganho de massa muscular. Nesse trabalho descritivo, foi analisado e selecionado fichas de pacientes com pós-operatório de ressecção de cabeça e colo femoral, em processo de reabilitação em um hospital veterinário escola, com o objetivo de realizar um estudo retrospectivo e relatar a recuperação funcional, tempo de recuperação e modalidades terapêuticas utilizadas nos cães selecionados. Dos 11 animais selecionados, 63.63\% obtiveram recuperação funcional em até um mês de reabilitação. Os animais que obtiveram uma recuperação em até um mês, 28,6\% realizaram cinco modalidades terapêuticas, 28,6\% três modalidades e $42,8 \%$ quatro modalidades, sendo que nenhum cão realizou todas as modalidades escolhidas neste estudo. A laserterapia foi o único método utilizado em todos os pacientes tratados. Conclui-se que a média de recuperação total de um cão em processo de Reabilitação Funcional pós procedimento de ressecção de cabeça e colo femoral, independente do diagnóstico anterior a cirurgia e diferentes modalidades terapêuticas utilizadas para cada paciente, é de um mês, sendo avaliado de acordo com o apoio total do membro, melhora da dor, melhora da claudicação e ganho de massa muscular. Sugere-se a realização de estudos controlados, com modalidades isoladas ou com o mesmo protocolo de tratamento, para que se possa avaliar se há diferenças de recuperação entre as diferentes formas de tratamento.

Palavras-chave: Ressecção da cabeça, colo femoral, reabilitação animal, fisiatria

\section{Functional recovery of dogs undergoing rehabilitation after femoral head and neck resection}

Abstract. The surgical procedure of resection of the femoral head and neck is a technique frequently used for degenerative and traumatic conditions that affect the coxofemoral joint. In the surgical postoperative period, rehabilitation is relevant for the patient to have pain control, full limb support, improvement in lameness and gain in muscle mass. In this descriptive work, records of patients with postoperative resection of the femoral head and neck, undergoing rehabilitation at a veterinary teaching hospital, were analyzed and selected, with the aim of conducting a retrospective study and reporting functional recovery, recovery time and therapeutic modalities used in selected dogs. Of the 11 selected animals, $63.6 \%$ achieved functional recovery within one month of rehabilitation. Of the animals that recovered within one month, $28.6 \%$ performed five therapeutic modalities, $28.6 \%$ three modalities and $42.8 \%$ four modalities, and no dog performed all the modalities chosen in this study. Laser therapy was the only method used in all treated patients. It is 
concluded that the average total recovery of a dog in the process of Functional Rehabilitation after the femoral head and neck resection procedure, regardless of the diagnosis prior to surgery and the different therapeutic modalities used for each patient, is one month, being evaluated from according to full limb support, pain improvement, lameness improvement and muscle mass gain. It is suggested that controlled studies be carried out, with isolated modalities or with the same treatment protocol, in order to assess whether there are differences in recovery between the different forms of treatment.

Keywords: Femoral head, neck resection, animal rehabilitation, physiatry

\section{Introdução}

A articulação coxofemoral em cães e gatos pode ser acometida por diferentes tipos de lesões. As principais afecções são degenerativas (displasia coxofemoral, necrose asséptica da cabeça do fêmur) ou traumáticas (luxações coxofemorais e fraturas de cabeça e colo femoral) (Barbosa et al., 2012).

O tratamento adequado difere do tipo de afecção e das alterações encontradas e pode ser conservativo ou cirúrgico. Dentre as várias técnicas cirúrgicas podemos citar algumas como: sinfisiodese púbica juvenil, osteotomia tripla da pelve, denervação acetabular seletiva, ressecção de cabeça e colo femoral e próteses de quadril. Já com relação ao tratamento conservativo ou pós cirúrgico podemos utilizar várias técnicas como: acupuntura, exercícios passivos e/ou assistidos, exercícios ativos, ultrassom terapêutico, laserterapia, nutracêuticos, analgésicos, anti-inflamatórios, magnetoterapia e eletroterapia, com intuito de promover analgesia, ganho de massa muscular, estimular apoio, melhora da amplitude de movimento do membro, reduzir impactos/sobrecarga em membros torácicos e coluna vertebral (Sharon \& Eztala, 2018; Sprada \& Minto, 2018).

Uma das técnicas cirúrgicas que traz bons resultados para as afecções de displasia coxofemoral, necrose asséptica da cabeça do fêmur, luxação coxofemoral e fraturas de cabeça e colo femoral, é a ressecção da cabeça e colo femoral, que é um procedimento de salvamento da função do membro pélvico afetado, aliviando também a dor associada a osteoartrose (Figura 1). O resultado dessa técnica pode não ser tão eficiente em casos de atrofia muscular muito evidente e/ou em animais com mais acima de $20 \mathrm{~kg}$ (Sprada \& Minto, 2018). O alívio da dor se dá, pois, a cabeça e colo femoral são retirados, eliminando o contato entre o acetábulo e a cabeça femoral, permitindo com que no lugar desse espaço seja criado um tecido cicatricial, uma pseudoartrose (Off \& Matis, 1997; Rocha et al., 2008; Souza \& Tudury, 2003).

Para realização da técnica é feito um acesso cranial lateral da articulação coxofemoral e então feito uma osteotomia do colo do fêmur, a linha de osteotomia se inicia na face medial do trocânter maior, com término na face proximal do trocânter menor. Caso o ligamento da cabeça femoral esteja intacto é feita sua incisão antes da osteotomia, para que seja possível realizar a rotação externa do fêmur em $90^{\circ}$ e luxação da cabeça femoral do acetábulo para facilitar a osteotomia. Após a remoção da cabeça e colo femoral é necessário examinar a porção restante do fêmur proximal, para verificar irregularidades ou porções remanescentes e se verificado a presença é necessário removê-las (Olmstead, 2019) (Figura 2).

O bom resultado dessa técnica pode ser influenciado por vários fatores, sendo os mais relevantes: técnica cirúrgica, idade do paciente, atrofia muscular e peso corpóreo. Animais com o peso corpóreo menor que $17 \mathrm{~kg}$, jovens e com uma melhor massa muscular antes do procedimento cirúrgico tendem a responder a cirurgia de uma forma mais eficiente (Ferrigno et al., 2004; Off \& Matis, 1997; Rocha et al., 2008; Souza \& Tudury, 2003).

Após o procedimento podemos observar algumas alterações como: encurtamento do membro, redução do ângulo de abdução e extensão do membro, desconforto após exercício excessivo, hipotrofia muscular e luxação de patela (Ferrigno et al., 2004; Off \& Matis, 1997; Rocha et al., 2008; Souza \& Tudury, 2003).

Existem diversas técnicas para a reabilitação pós-operatória, e dentre elas estão acupuntura, ultrassom terapêutico, laserterapia, corrente interferencial, cinesioterapia e hidroterapia.

A acupuntura faz parte da Medicina Tradicional Chinesa (MTC), que trata o equilíbrio energético corporal. Na MTC a doença é um desequilíbrio e o corpo é uma estrutura integrada e energética, onde uma doença se instala quando algo impede o fluxo energético (Xie \& Preast, 2012). 
Tanto a MTC quanto a medicina ocidental são utilizados testes para diagnósticos, porém na MTC os testes ocorrem pela palpação de pulso, pontos Shu e Mu (sensibilidade desses pontos está ligada a desequilíbrio nos órgãos internos correspondentes) e após o diagnóstico é feito o tratamento com acupuntura, fitoterápicos ou outras técnicas ocidentais necessárias (Xie \& Preast, 2012). A MTC é muito utilizada de modo preventivo ou também em doenças crônicas, onde especialmente a medicina ocidental pode apenas controlar e não curar (Xie \& Preast, 2012).

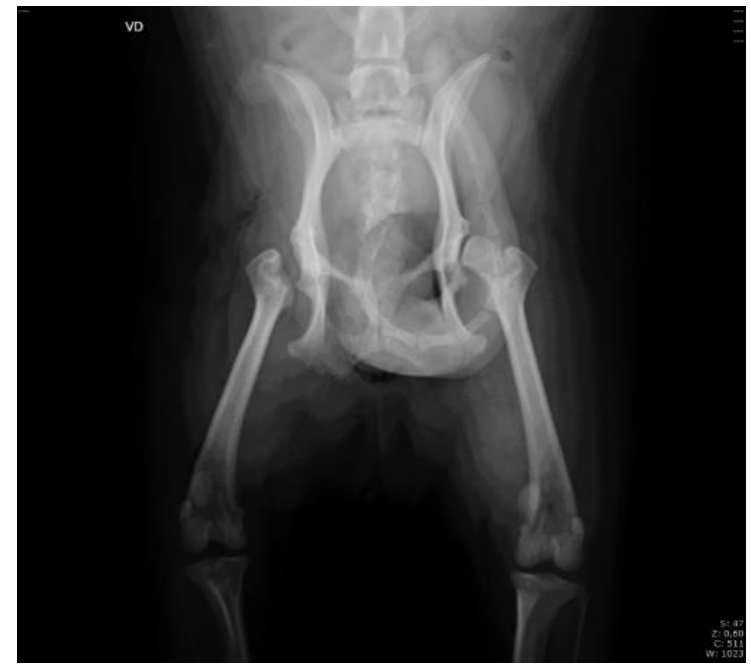

Figura 1. Radiografia em posição ventro-dorsal de pós-operatório de ressecção de cabeça e colo femoral em um cão. Fonte: Arquivo Hospital Anhembi Morumbi (2020).

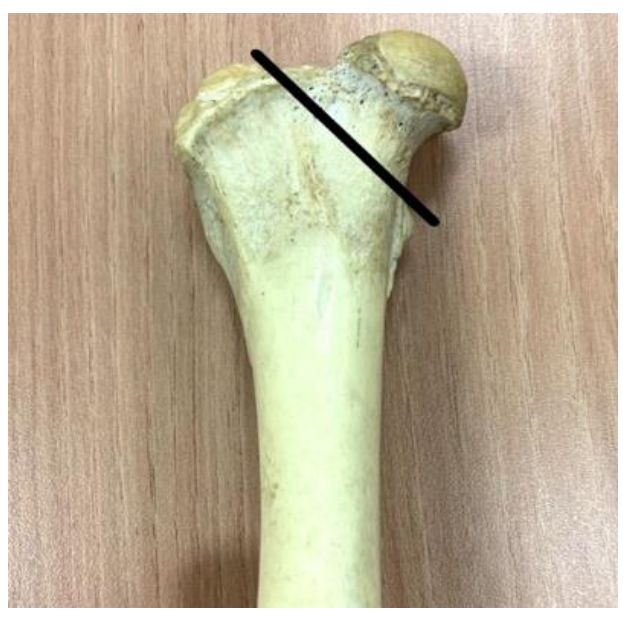

Figura 2. Sítio de osteotomia para ressecção de cabeça e colo femoral.

Os pontos de acupuntura são locais específicos de sensibilidade espontânea ao estímulo e à resistência elétrica, é uma área de alta condutividade elétrica. Esses acupontos são próximos de articulações, bainhas tendíneas, vasos, nervos e regiões de penetração de feixes nervosos da pele, quando estimulados ativam o sistema modulador da dor e homeostático (Deadman et al., 2001; Taffarel \& Freitas, 2009).

O ultrassom se refere a ondas acústicas de alta frequência, cuja frequências são superiores a $20 \mathrm{KHz}$ (Steiss \& Levine, 2005). As ondas ultrassônicas são aplicadas de duas formas conhecidas como contínuo e pulsado e o que difere um da outra é a interrupção da propagação de energia. O modo contínuo não existe a interrupção da aplicação de energia sobre os tecidos, fazendo com que haja aquecimento dos tecidos submetidos. Já no modo pulsado existe uma breve interrupção na propagação de energia, resultando em uma redução de aquecimento tecidual e um maior efeito mecânico (Hummel et al., 2019).

Os efeitos produzidos pela ação do ultrassom em tecidos biológicos dependem da interação de diversos fatores como, intensidade $\left(0,1\right.$ a $\left.3 \mathrm{~W} / \mathrm{cm}^{2}\right)$, frequência ( $1 \mathrm{Mhz}$ a $\left.3 \mathrm{Mhz}\right)$, tempo de exposição e estado fisiológico do tecido (Hummel et al., 2019).

Os efeitos térmicos produzidos pelo modo contínuo incluem: aumento da extensibilidade do colágeno, analgesia, melhora de processos inflamatórios crônicos, diminuição de espasmos musculares (Steiss \& Levine, 2005). No modo pulsado os efeitos mais prevalentes são os mecânicos, que incluem: estimulação de deposição de colágeno, regeneração de tecidos, reparo ósseo, ativação de ciclo de cálcio, proliferação de fibroblastos, redução de edema (Steiss \& Levine, 2005). A laserterapia está dentro da fototerapia, nessa terapia é convertido a energia elétrica em uma energia eletromagnética lumínica (Hummel et al., 2019).

O laser é classificado de acordo com o risco de causar lesão ocular, sendo classificados de I a IV, as classes IIIB e IV são terapêuticas (Hummel et al., 2019). A luz laser é monocromática, coerente e colimada, fazendo com que haja uma maior penetração e absorção de energia em tecidos mais profundos (Hummel et al., 2019).

Existem diversos parâmetros físicos que são necessários para obter respostas positivas através da terapia , que são: Potência (demanda energética do aparelho, medida em Watts ou Milliwatts, podendo ir de $5 \mathrm{nW}$ a $15 \mathrm{~W}$, a potência do aparelho determina a velocidade de aplicação); Comprimento de onda 
(profundidade de penetração da luz, expressada em nanômetros e a faixa mais utilizada é de 600 a 980 $\mathrm{nm}$ ), dosagem (densidade energética depositada no tecido, calculada em Joules e compreendida entre 1 a $20 \mathrm{~J} / \mathrm{cm}^{2}$, esta dosagem pode ser interferida de acordo com o objetivo do tratamento, cor de pele/pelo e tamanho do animal (Hummel et al., 2019).

Os efeitos biológicos do laser são diversos, alguns deles são: analgesia (estimula aumento de serotonina, endorfinas beta e óxido nítrico, normaliza canais iônicos, aumenta potencial de ação de ação das células nervosas, bloqueia a despolarização dos nervos aferentes de fibra C), anti-inflamatório (diminui a síntese de prostaglandinas inflamatórias, redução de interleucina-1, diminui o nível de proteína $\mathrm{C}$ reativa, acelera a atividade leucocitária, estimula fluxo linfático, aumenta a angiogênese) reparação tecidual (induz aumento da atividade de macrófagos e fibroblastos, estimula neovascularização e proliferação de queratinócitos, induz uma epitelização precoce, aumenta níveis fatores de crescimento, aumenta o nível de ATP) regeneração nervosa, consolidação óssea (Hummel et al., 2019; Pryor \& Millis, 2015).

A eletroterapia é uma técnica onde é aplicado uma corrente elétrica, usada para uma variedade de fins, incluindo aumento de força muscular, controle de dor aguda ou crônica, patologias musculares ou ortopédicas, recuperação de fraturas, entre outras (Hummel et al., 2019; Steiss \& Levine, 2005). Podemos dividir as correntes de eletroterapia em dois grupos, os que são usados para fortalecimento e reeducação muscular e os que são responsáveis para analgesia (Steiss \& Levine, 2005). As estimulações elétricas que promovem contração muscular são EMS (estimulação elétrica muscular) e NMES (estimulação elétrica neuromuscular) e correntes que fazem bloqueios de terminações nervosas sensitivas para alívio de dor são TENS (estimulação elétrica nervosa transcutânea), IFC (corrente interferencial) (Sanches \& Assis, 2018).

A hidroterapia é uma técnica da reabilitação que proporciona vários benefícios para um animal em reabilitação (Hummel et al., 2019). A água apresenta propriedades físicas como densidade relativa, pressão hidrostática, força de empuxo, força de arrasto e resistência, tensão superficial, viscosidade, impulso e temperatura, que são importantes para a recuperação funcional do animal, alívio da dor na movimentação, redução de peso/ estresse em articulações, redução de edemas, fortalecimento de músculos, apoio de membros, amplitude de movimento de articulações, entre outros (Dycus et al., 2017; Hummel et al., 2019). As modalidades mais utilizadas são natação e esteira aquática, quanto mais alto o nível da água, menor será o estresse causado nas articulações. Com água ao nível do maléolo lateral da fíbula, os cães suportam cerca de $91 \%$ do peso corporal. Com água no côndilo lateral do fêmur, os cães sustentam $85 \%$ de seu corpo peso. Com água para o trocanter maior, os cães sustentam apenas $38 \%$ de seu peso corporal (Dycus et al., 2017).

A cinesioterapia é a prática de exercícios terapêuticos dentro da fisioterapia, que são usados para obtermos uma melhor amplitude de movimento articular, melhor alongamento, aumento de força e massa muscular, melhor condicionamento, apoio de membro, estímulo de propriocepção, entre outros (Drum et al., 2015). Os exercícios podem ser praticados de várias formas, apenas com as mãos como uma amplitude passiva de movimento e o alongamento, podem ser feitos utilizando bolas, cones, pranchas de propriocepção, obstáculos e exercícios ativos que não precisam de nenhum equipamento (Drum et al., 2015).

Nesse trabalho descritivo, será analisado e selecionado fichas de pacientes com pós-operatório de ressecção de cabeça e colo femoral, em processo de reabilitação, com o objetivo de realizar um estudo retrospectivo e relatar a recuperação funcional, tempo de recuperação e modalidades terapêuticas utilizadas nos cães selecionados.

\section{Material e métodos}

Foi utilizado uma metodologia de pesquisa descritiva, fazendo um levantamento, análise e seleção de fichas de pacientes caninos da área de Reabilitação Veterinária em um hospital escola, no período de 2013 a 2020, que foram submetidos ao processo de reabilitação pós ressecção de cabeça e colo femoral, devido displasia coxofemoral (DCF), luxação coxofemoral (LCF), fratura da cabeça e colo femoral (FCCF) e necrose asséptica da cabeça femoral (NACF), sem descriminação de raça, sexo, idade e peso corporal. 
Após análises de fichas, foram selecionados 11 cães, que apresentavam sintomatologia semelhante, dor, não apoio do membro, apoio intermitente e/ou claudicação.

Os cães selecionados foram submetidos às técnicas de acupuntura ( $\mathrm{ACP})$, ultrassom terapêutico, laserterapia, eletroterapia, cinesioterapia e hidroterapia.

Localização e pontos de acupuntura mais utilizados nos animais submetidos a este trabalho:

VB30: Na depressão, a meio caminho entre o trocânter maior e o tubérculo ísquio (tubérculo isquiático). VB29: Na depressão, 1/3 da distância do trocânter maior à espinha ilíaca dorsal cranial (tuberosidade sacral).

B54: Na depressão dorsal ao trocanter maior.

B40: Na depressão da fossa poplítea no ponto médio da prega transversa, na divisão do bíceps femoral e do semitendíneo $\mathrm{mm}$. Esse ponto é encontrado mais facilmente com o joelho flexionado.

B26: Na depressão 1,5 cun (unidade de mensuração de acupuntura do corpo) lateral à linha média dorsal ao nível do espaço lombossacral.

Bai hui: Na depressão na linha média dorsal no espaço lombossacral.

\section{Resultados e discussão}

Após a análise das fichas, foi constatado que os diagnósticos com maior predominância são de animais com displasia coxofemoral e/ou luxação coxofemoral (63,6\%). Três animais $(27,3 \%)$ foram submetidos à acupuntura, seis $(54,5 \%)$ utilizaram eletroterapia, nove $(82,0 \%)$ utilizaram Ultrassom Terapêutico, nove $(82,0 \%)$ utilizaram hidroterapia, dez $(91,0 \%)$ foram submetidos à cinesioterapia e onze $(100 \%)$ cães utilizaram laserterapia.

Cinco animais $(45,4 \%)$ apresentavam seis meses a um ano de idade, três $(27,3 \%)$ cães com idades de dois a quatro anos e três $(27,3 \%)$ cães entre nove e treze anos. Com relação ao porte dos animais $91 \%$ (10), eram de porte pequeno e as raças de maior prevalência foram o Spitz Alemão e Poodle.

A recuperação total foi avaliada devido a melhora da dor, apoio do membro acometido, melhora da claudicação e recuperação de massa muscular.

Conforme a avaliação dos resultados encontrados no presente estudo, sugere-se que sete $(63,63 \%)$ dos cães obtiveram recuperação total por volta de vinte dias a um mês de tratamento, três $(27,27 \%)$ animais por volta de um mês e vinte dias e dois meses e apenas um $(9,1 \%)$ cão obteve alta em três meses.

Os animais que obtiveram uma recuperação mais rápida, entre vinte dias a um mês de tratamento, $28,6 \%$ realizaram cinco modalidades, $28,6 \%$ três modalidades, $42,8 \%$ quatro modalidades, sendo que nenhum cão realizou todas as modalidades e a laserterapia foi o único método que todos neste grupo utilizaram.

Tabela 1. Número de animais, idade, raça, diagnóstico, técnicas utilizadas e o tempo de recuperação (apoio total de membro, sem claudicação e sem dor em região coxofemoral) de cada paciente em tratamento

\begin{tabular}{|c|c|c|c|c|c|c|}
\hline $\begin{array}{l}\text { Animal, } \\
\mathrm{N}^{\mathrm{o}}\end{array}$ & Idade & Raça & Diagnóstico & $\begin{array}{c}\mathrm{N}^{0} \text { de técnicas } \\
\text { utilizadas }\end{array}$ & $\mathrm{N}^{\mathrm{o}}$ de sessões & $\begin{array}{c}\text { Tempo de } \\
\text { recuperação }\end{array}$ \\
\hline 1 & 10 meses & Spitz Alemão & NACF & 6 & 9 sessões & 2 meses \\
\hline 2 & 4 anos & Pug & DCF & 4 & 7 sessões & 1 mês \\
\hline 3 & 2 anos & Yorkshire & $\mathrm{LCF}$ & 3 & 4 sessões & 1 mês \\
\hline 4 & 9 anos & Poodle & DCF & 4 & 5 sessões & 1 mês \\
\hline 5 & 4 anos & Border Collie & $\mathrm{DCF}$ & 5 & 21 sessões & 3 meses \\
\hline 6 & 6 meses & SRD & $\mathrm{FCCF}$ & 5 & 7 sessões & 2 meses \\
\hline 7 & 1 ano e 2 meses & SRD & $\mathrm{LCF}+\mathrm{DCF}$ & 5 & 4 sessões & 1 mês \\
\hline 8 & 1 ano & $5,7 \mathrm{~kg}$ & NACF & 4 & 4 sessões & 1 mês \\
\hline 9 & 9 anos & Poodle & LCF & 3 & 4 sessões & 1 mês \\
\hline 10 & 13 anos & Lhasa Apso & LCF & 4 & 8 sessões & 1 mês e 20 dias \\
\hline 11 & 10 meses & Spitz Alemão & NACF & 5 & 4 sessões & 20 dias \\
\hline
\end{tabular}

DCF: Displasia coxofemoral; NACF: Necrose asséptica da cabeça femoral; FCCF: Fratura da cabeça e colo femoral; LCF: Luxação coxofemoral.

Em comparação com o relato de caso de Silva (2016), onde o animal submetido a reabilitação pós ressecção de cabeça e colo femoral era da raça Pastor Alemão, onde foram utilizados laserterapia, magnetoterapia, fototerapia, ozonioterapia, eletroterapia, moxaterapia, acupuntura, hidroesteira e 
Haihua. Foi necessário pausar a reabilitação na terceira sessão, pois o animal precisou ser submetido a um procedimento cirúrgico de reparação decorrente a complicações da colocefalectomia. No retorno ao processo de reabilitação após o segundo procedimento, o animal começou a não apresentar mais dor em região coxofemoral na sua nona sessão, porém não foi exposto o tempo que foi percorrido até a nona sessão no animal.

Segundo Anunciação et al. (2017), em seu relato de caso de uma cadela da raça West Highland White Terrier, que foi submetida a fisioterapia após procedimento de ressecção de cabeça e colo femoral, onde foi utilizado laserterapia, hidroterapia, eletroterapia e cinesioterapia, o animal obteve recuperação total do quadro após a quarta semana de tratamento.

Em contraste com o estudo de Sterin \& Rebrij (2006), onde foram analisados quatro casos de animais foram submetidos à cirurgia de ressecção de cabeça e colo femoral, devido três animais apresentarem displasia coxofemoral e um deles com luxação traumática. Foram utilizadas as modalidades de laserterapia, magnetoterapia, eletroterapia e cinesioterapia. Neste estudo segundo os escritores, todos os animais obtiveram recuperação total após 30 a 45 dias, sendo avaliado dor, ganho de massa muscular e poio de membro.

Em estudo retrospectivo em 129 animais de pequeno porte que foram submetidos à ressecção de cabeça e colo femoral, feito por Barbosa et al. (2019), apenas 9,3\% apresentaram melhora total da claudicação com até um mês pós cirurgia, porém não foi informado se algum animal foi submetido a alguma técnica de reabilitação.

\section{Conclusão}

A média de recuperação total de um cão em processo de Reabilitação Funcional pós procedimento de ressecção de cabeça e colo femoral, independente do diagnóstico anterior a cirurgia e diferentes modalidades terapêuticas utilizadas para cada paciente, é de um mês, sendo avaliado de acordo com o apoio total do membro, melhora da dor, melhora da claudicação e ganho de massa muscular evidente. Sugere-se a realização de estudos controlados, com modalidades isoladas ou com o mesmo protocolo de tratamento, para que se possa avaliar se há diferenças de recuperação entre as diferentes formas de tratamento.

\section{Referências bibliográficas}

Anunciação, A. A., Souza, J. C., Silva, L. E., \& Alcântara, M. A. (2017). Uso da fisioterapia no pósoperatório de cão submetido a colocefalectomia - Relato de caso. Revista Eletrônica Biociências, Biotecnologia e Saúde, 10(19), 142-144.

Barbosa, A. L. T., Schossler, J. E. W., Bolli, C. M., Lemos, L. F. C., \& Medeiros, C. (2012). Recuperação funcional coxofemoral pós-operatória em cães: estudo clínico, radiográfico e biomecânico. Ciência Rural, 42, 2011-2017. https://doi.org/10.1590/S0103-84782012005000099.

Deadman, P. J. of C. M. P., Baker, K., \& Al-Khafaji, M. (2001). A Manual of acupuncture. : : Journal of Chinese Medicine Publications, 376-380.

Drum, M. G., Marcellin-Little, D. J., \& Davis, M. S. (2015). Principles and applications of therapeutic exercises for small animals. Veterinary Clinics: Small Animal Practice, 45(1), 73-90. https://doi.org/10.1016/j.cvsm.2014.09.005.

Dycus, D. L., Levine, D., \& Marcellin-Little, D. J. (2017). Physical rehabilitation for the management of canine hip dysplasia. Veterinary Clinics: Small Animal Practice, 47(4), 823-850. https://doi.org/10.1016/j.cvsm.2017.02.006.

Ferrigno, C. R. A., D’Avila, R. S., Yamamoto, E. Y., Yazbek, K. V. B., \& Ferraz, V. C. M. (2004). Estudo da técnica de denervação da cápsula articular coxofemoral no tratamento da dor em cães com displasia coxofemoral: resultados preliminares. Brazilian Journal of Veterinary Research and Animal Science, 41, 169-170. https://doi.org/10.11606/issn.2318-3659.v41isupl.p169-170.

Hummel, J., Vicente, G., Formenton, M. R., \& Cadini, B. M. (2019). Tratado de fisioterapia e fisiatria de pequenos animais. Editora Payá. 
Off, W., \& Matis, U. (1997). Excision arthroplasty of the hip joint in dogs and cats. Clinical, radiographic and gait analysis findings at the surgical veterinary clinic of the Ludwig Maximilians University of Munich. Tierarztliche Praxis, 25(4), 379-387. https://doi.org/10.1055/s-00371617478.

Olmstead, M. L. (2019). Anormalidades da articulação coxofemoral. In S. J. Birchard \& R. G. Sherding (Eds.), Manual Saunders Clínica de Pequenos Animais (pp. 1134-1141). Editora Roca.

Pryor, B., \& Millis, D. L. (2015). Therapeutic laser in veterinary medicine. Veterinary Clinics: Small Animal Practice, 45(1), 45-56. https://doi.org/10.1016/j.cvsm.2014.09.003.

Rocha, F. P. C., Silva, D., Benedette, M. F., Santos, D. A. N., Costa, E. A. A., \& Dias, L. G. G. G. (2008). Displasia coxofemoral em cães. Revista Científica Eletrônica de Medicina Veterinária, 4(11), 1-7.

Sanches, M., \& Assis, L. (2018). Eletroterapia. In R. S. Lopes \& R. Diniz (Eds.), Fisiatria em pequenos animais (pp. 92-102). Editora Inteligente.

Sharon, L., \& Eztala, S. (2018). Fisiatria em pequenos animais. Editora Inteligente.

Silva, I. T. C. P. (2016). Displasia coxofemoral e tratamento fisioterápico pós colocefalectomia: relato de caso. Universidade Federal de Porto Alegre.

Souza, A. F. A., \& Tudury, E. A. (2003). Displasia coxofemoral: diagnóstico clínico e radiográfico: revisão. Clínica Veterinária, 8(47), 54-66.

Sprada, A. ., \& Minto, B. W. (2018). Afecções do quadril. In R. . Lopes \& R. Diniz (Eds.), Fisiatria em pequenos animais (pp. 360-380). Editora Inteligente.

Steiss, J. E., \& Levine, D. (2005). Physical agent modalities. Veterinary Clinics: Small Animal Practice, 35(6), 1317-1333.

Sterin, G. M., \& Rebrij, T. A. (2006). Tratamento fisioterapêutico postquirúrgico empleado en la exeresis de la cabeza y cuello femoral. . Congresso Vemevepa.

Taffarel, M. O., \& Freitas, P. M. C. (2009). Acupuncture and analgesia: clinical applications and main acupoints. Ciência Rural, 39(9), 2665-2672. https://doi.org/10.1590/S0103-84782009000900047.

Xie, H., \& Preast, V. (2012). Medicina veterinária tradicional chinesa: princípios fundamentais. Medvep.

Histórico do artigo:

Recebido: 29 de setembro de 2021

Aprovado: 7 de novembro de 2021

Disponível online: 3 de janeiro de 2022
Licenciamento: Este artigo é publicado na modalidade Acesso Aberto sob a licença Creative Commons Atribuição 4.0 (CC-BY 4.0), a qual permite uso irrestrito, distribuição, reprodução em qualquer meio, desde que o autor e a fonte sejam devidamente creditados. 